\title{
Embodied Cognition: Knowing in the Head is Not Enough
}

\section{Michelle Daniel, Eric Wilson, Dario Torre, Steven J Durning, Valerie Lang}

In embodied cognition the mind, body and environment are interconnected and interdependent for both making diagnoses and enacting management plans, through perception-action loops mediated by cognition. ${ }^{1}$ This is true regardless of physician specialty but is particularly evident in procedural fields, with significant implications for teaching and assessment.

Consider the following: An American-trained family physician is called to deliver a baby at a rural clinic in Mexico. The patient is a 17year-old, first time mom, and based on palpation, the baby is quite large. After delivery of the head, the physician sees that the baby's face appears plethoric and the head appears to be "turtling" back into the birth canal. The physician recognizes a shoulder dystocia, performs an episiotomy, and calls for the nurse to lift the legs in a McRobert's maneuver (1) while applying suprapubic pressure (2). These measures are unsuccessful. The physician knows next steps are either to deliver the posterior arm or to break the clavicle, but he doesn't know what that feels like, resulting in actions that are ineffective. The local obstetrician arrives, breaks the clavicle, and delivers an apneic baby.

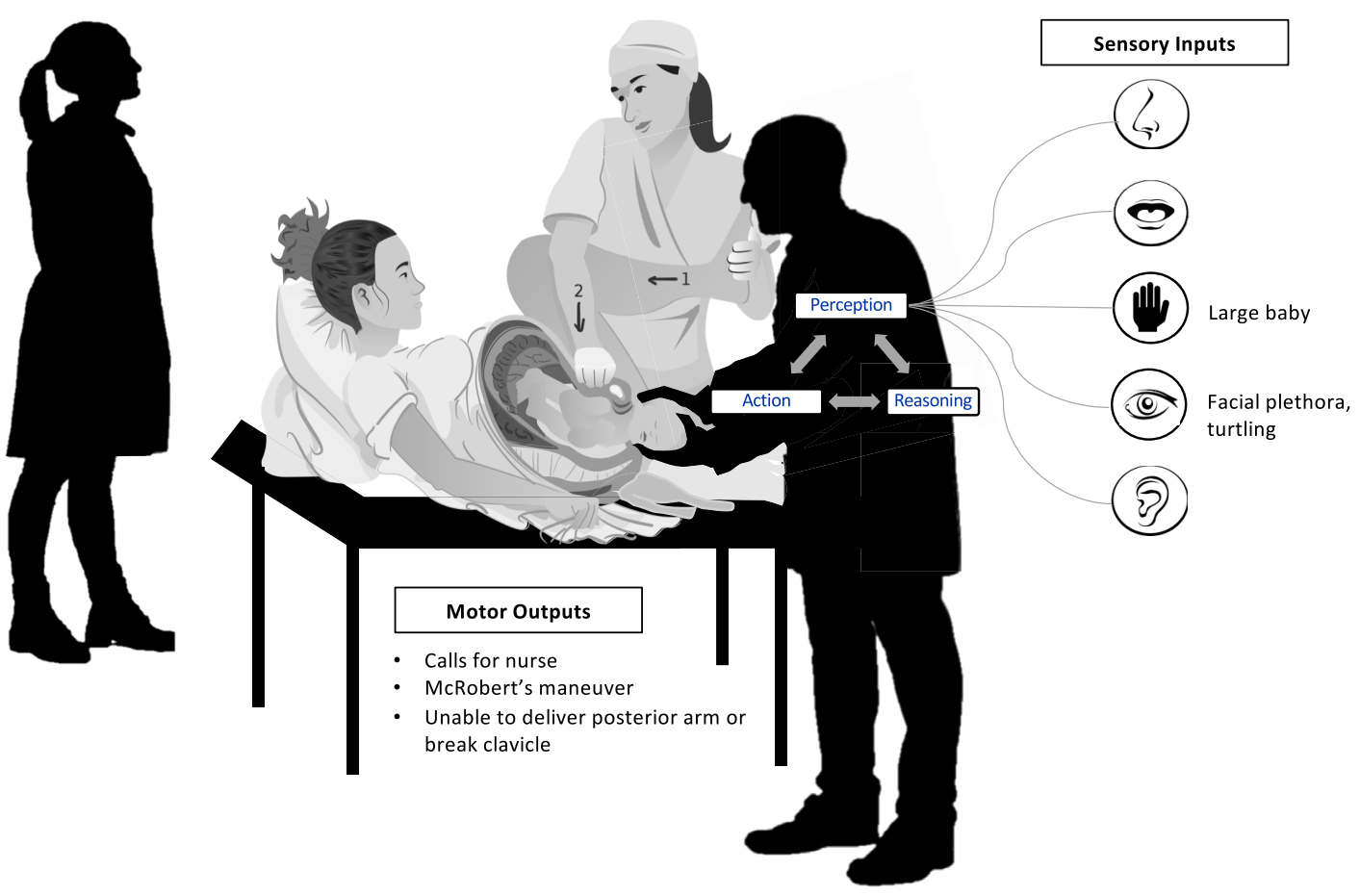

In this example, based on sensory inputs (i.e. palpating a large baby, seeing facial plethora and "turtling"), the American physician identifies a shoulder dystocia and takes action (i.e., he calls for the nurse and asks for a McRobert's maneuver.) When these actions fail, he knows what to do next, but lacks the sensorimotor experience of the Mexican obstetrician to successfully intervene in this labor complication (i.e., he couldn't locate the posterior arm or achieve the proper leverage to break the clavicle). The case illustrates that knowing "in the head" is not enough. Physicians must have the opportunity to link their perceptions and cognitive knowledge with the motor capabilities needed to execute a specific task. American family physicians typically learn about shoulder dystocia from studying textbooks or reading paper cases. Obstetricians are more likely to learn about shoulder dystocia through simulations (in the United States) or direct experience with multiple complicated deliveries (in Mexico). Their "knowledge" is embodied (i.e., their cognitive processes are organized into conceptual frameworks entangled with past perceptions and actions in memory) and they have thus developed a rich sensorimotor repertoire to manage this crisis. 
*Corresponding author: Michelle Daniel, Office of Medical Student Education, University of Michigan Medical School, 6123 Taubman Health Sciences Library, 1135 Catherine, Ann Arbor, 48109-0624, Michigan, USA, E-mail: micdan@umich.edu

Eric Wilson: Medical Student, University of Michigan Medical School, Ann Arbor, MI, USA

Dario Torre and Steven J. Durning: Internal Medicine, Uniformed Services University of the Health Sciences, Bethesda, MD, USA
Valerie Lang: University of Rochester School of Medicine \& Dentistry, Rochester, NY, USA

https://doi.org/10.1515/dx-2020-0004

Published online June 19, 2020

Supplementary Material: The licenses for the images are provided in the online version of this article (https://doi.org/10.1515/dx-2020-0004). 